\title{
Self-cleaning photonic coatings
}

\author{
Ronald Pirich, John Weir, Dennis Leyble, and \\ Michael DiGiuseppe
}

A new family of coating systems provides self-decontamination against dust and biological pathogens for future space exploration.

Dust contamination is a serious problem for equipment and vehicles for space exploration. For example, the Moon's dust (lunar regolith) is chemically composed of several elements and compounds, and lunar 'weathering' has left the soil with a relatively fine texture compared to the size distribution of terrestrial dust particles. Previous NASA investigations indicated a lunar-regolith deposition rate of approximately $0.3 \%$ coverage per day, although this will vary both geographically and as a function of time. In addition, the Moon's surface is electrostatically charged by its interaction with the local plasma environment and solar UV- and x-ray-induced photoemission of electrons. The lunar thermal environment poses unique challenges to coatings since it is characterized by large temperature variations, long hot and cold soak times, and reduced heat-rejection capability caused by the presence of regolith. Dust gathers on photonic sensors, inhibiting motion and data gathering. Devices that require transparency to light for maximum efficiency (such as solar photovoltaic power systems, video cameras, and optical or IR detectors) also suffer from dust accumulation (see Figure 1). Some scientists have even suggested another potential hazard, that equipment might inadvertently capture extraterrestrial bacteria or spores, possibly leading to catastrophic contamination of human habitats.

The Apollo astronauts noted that their extravehicularmobility suits were worn through the outer layer. Gauge dials were so abraded during the Apollo 16 mission that they were unreadable. The sun shade on Harrison Schmitt's face plate was so scratched that he could not see in certain directions. NASA strongly believes that these abrasion problems must be solved, especially for very light-transmission-sensitive photonic lenses and coatings. Dust contaminant particles can absorb or scatter light either out of or into certain pixels, thus giving rise to dark and bright artifacts, respectively.

Only very localized and weak magnetic fields exist on the lunar surface, leaving it essentially directly exposed to the impact
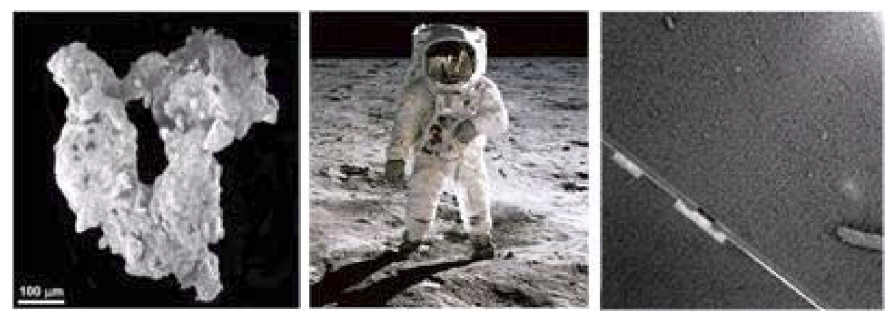

Figure 1. Examples of (left) a lunar-dust contaminant particle, and contamination-critical surfaces including (middle) extravehicularactivity suits and (right) solar panels attached to NASA's Spirit rover. ${ }^{1,2}$

of solar UV and x-rays, solar-wind plasma, and energetic particles. The lack of an atmosphere results in positive (negative) charging of the environment in sunlight (shadow) regions. This leads to potentials that can vary over orders of magnitude in response to changing solar illumination and plasma conditions. At the macroscale, contaminant adhesion caused by surface (van der Waals) forces are very small and can be easily perturbed by other external forces. However, on nanoscales van der Waals forces can be significant. In general, ionizing radiation does not affect these intermolecular forces unless the surface-molecular structure is changed, since van der Waals interactions are always present between molecules. They only depend on the number of electrons in a given molecule and the distance between molecules. Ionizing radiation would primarily affect the surface chemistry of the material used for the coating material.

In cooperation with NASA, small businesses, and leading universities, we are developing a family of self-cleaning and anti-contamination coating systems that provide selfdecontamination against dust, chemical agents, and biological pathogens, as well as toxic industrial compounds and materials and other environmental foulants. They are selfcleaning because contaminants are easily removed from surfaces by solar wind or mechanical vibration. More importantly for lunar-dust mitigation, we have shown that these coatings can be designed using low-surface-energy materials that reduce all particle adhesion very significantly (see Figure 2). In addition, we are developing a family of hybrid catalytic 


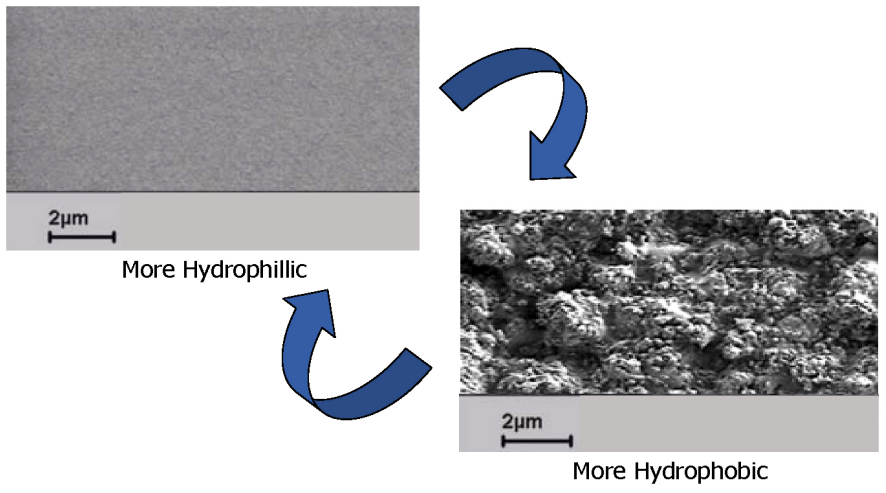

Figure 2. We are applying techniques for tailoring coating morphologies and developing a family of self-cleaning and anti-contamination coating systems for a variety of photonic applications including sensors, photovoltaics, and thermal radiators for future space exploration. ${ }^{3}$

coatings that present a unique and paradigm-changing approach to dust mitigation using biocidal and chemical neutralization features. Dust accumulation on sensors, solar cells, and thermal radiators gradually reduces their effectiveness until they no longer generate or dissipate enough energy to sustain operation of equipment. Our new coating systems have the potential to minimize these effects. NASA believes it is also critical to prevent accumulation of dust and other contaminants on vehicles and mechanical equipment to prevent performance degradation and reduce maintenance. These coatings offer the potential to simplify decontamination procedures by neutralizing unintentionally captured micro-organisms or harmful chemicals on surfaces of structures and equipment during assembly-both on earth and in extraterrestrial environments-preventing inadvertent and possibly catastrophic contamination.

We are designing an integrated approach to solving the dust problems-including the required coatings propertiesas a function of ionizing radiation, temperature, and spacecontamination effects. We are developing and testing hydrophilic, hydrophobic, and hybrid-coating self-cleaning techniques, as well as a new approach incorporating various catalytic mechanisms (stoichiometric, photo-, and electrocatalytic) for decontamination in the lunar environment.

Controlling and excluding dust particles from photonic systems will be of paramount importance to ensure the safety of future space exploration missions, reliable operation of solar arrays, radiators, sensors, internal/external structural surfaces, and other photonic-related equipment. Development of selfcleaning coatings to mitigate dust contamination accumulation is critical to meeting extended exploration mission requirements. One potential component of this solution includes a range of coatings that possess the required photonic performance and reduce extraterrestrial cleaning requirements, while providing lower maintenance and overall mission costs.

\section{Author Information}

Ronald Pirich, John Weir, Dennis Leyble, and Michael DiGiuseppe

Technology Development

Northrop Grumman Aerospace Systems

Bethpage, NY

Ronald Pirich has been at the Northrop Grumman Corporation since 1977. He is currently a technical fellow responsible for integration/systems engineering for electromagnetic environmental effects, chemical, biological, nuclear, radiological, and explosives warfare defense, next-generation sensors (e.g., terahertz), and advanced coatings.

John Weir has over 35 years experience in management and research into the qualification of materials for various aerospace applications. John received his BS and MS degrees from Stony Brook University and an MBA in finance from Long Island University. He holds an ISO 9000 certification from the International Organization for Standardization and is a registered professional engineer in New York and Pennsylvania.

Dennis Leyble is a research engineer at the Aerospace Systems Advanced Programs and Technology/Technology Development Center. Dennis has made significant contributions to several technology programs including space exploration. He graduated with a BS from Stony Brook University.

Michael DiGiuseppe leads the space surface-science program in areas critical to mission success in returning to the Moon as a first step in going to Mars and beyond. He received a BS in chemistry from Brooklyn Polytechnic Institute and a PhD in solidstate chemistry from Brown University. He is a member of the American Chemical Society, the American Association for Crystal Growth, the Optical Society of America, and SPIE. 


\section{References}

1. L. A. Taylor, C. M. Pieters, L. P. Keller, R. V. Morris, and D. S. McKay, Lunar mare soils: space weathering and the major effects of surface-correlated nanophase Fe, J. Geophys. Lett. 106, pp. 27985-27999, 2001.

2. D. K. Hollingsworth, L. C. Witte, and A. Higgins, University of Houston-Clear Lake, ISSO Annu. Rep., pp. 68-71, 2005.

3. R. Pirich, J. Weir, D. Leyble, and M. DiGiuseppe, The effects of ionizing radiation,

temperature and space contamination on photonic coatings, Proc. SPIE 7467. In press. 